\title{
Aplasia medular adquirida y embarazo. Caso clínico
}

\author{
PILAR LEÓN ${ }^{1, \mathrm{a}}$, JOSEFINA LERÍA ${ }^{2, \mathrm{~b}}$, DANIELA CARDEMIL ${ }^{1, \mathrm{a}}$, \\ ANGÉLICA DÍAZ ${ }^{2}$, MIGUELINA HERNÁNDEZ ${ }^{3}$, \\ MAGDALENA CHUBRETOVIC ${ }^{c}$, JOSEFA GONZÁLEZ ${ }^{c}$, \\ MARÍA ELENA CABRERA ${ }^{1}$
}

\section{Acquired aplastic anemia during pregnancy. Report of one case}

Aplastic anemia (AA) or acquired aplastic anemia is an uncommon and potentially fatal disease. It is defined as reduction of at least two peripheral blood series, associated with persistent bone marrow hypocellularity. It's association with pregnancy is even more uncommon, and it may result in high mortality for the mother and the child. We report an adolescent female with aplastic anemia, which worsened during pregnancy. Her hemoglobin was $5.2 \mathrm{~g} / \mathrm{dl}$, her leukocytes were 1,833/ul and her platelets were 19,000/ul. She was initially treated with cyclosporine and horse antithymocyte globulin without hematologic improvement. At 36 weeks of pregnancy methylprednisolone and platelet transfusions were used and a cesarean section was performed. Six month later she continues to require red blood cell and platelet transfusions.

(Rev Med Chile 2018; 146: 399-402)

Key words: Anemia, Aplastic; Immunosuppression; Pregnancy.
'Unidad Hematología, Hospital del Salvador. Santiago, Chile. ${ }^{2}$ Unidad de Alto Riesgo Obstétrico, Hospital Luis Tisné Brousse. Santiago, Chile. ${ }^{3}$ CRS Cordillera Oriente. Santiago, Chile.

aBecadas de Hematología Universidad de Chile, Hospital del Salvador.

becada de Ginecología y Obstetricia Universidad de Chile, Hospital Luis Tisné Brousse Interna de Medicina, Universidad de Chile.

Los autores declaran no tener concflictos de interés.

Trabajo no recibió finaciamiento.

Recibido el 3 de febrero de 2017 aceptado el 24 de abril de 2018.

Correspondencia a:

Pilar León Maldonado Francisco Sosa Cousiño 610, departamento 805, Concón, Valparaíso, Chile. pilarleonm@gmail.com

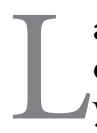

a anemia aplástica (AA) o aplasia medular o adquirida es una patología infrecuente $\int$ y potencialmente mortal. La incidencia en países occidentales es 2 casos por millón de habitantes. Se caracteriza por una disminución o ausencia de precursores hematopoyéticos en la médula ósea, lo cual se expresa con distintos grados de citopenias. Se define como disminución de al menos dos series en sangre periférica, asociada a hipocelularidad persistente en médula ósea $(<25 \%)^{1}$. Se clasifica como aplasia medular severa (AMS) con dos de los siguientes criterios (criterios de Camitta): 1) recuento absoluto de neutrófilos $($ RAN $)<500 /$ ul; 2) recuento de plaquetas $<20.000 /$ ul y recuento de reticulocitos corregido $<1 \%$ y muy severa (AMMS) si el RAN $<200 /$ ul $^{2}$. Se ha identificado una amplia variedad de drogas, químicos, virus y enfermedades autoinmunes como agentes etiológicos, sin embargo, $70 \%$ de los casos son idiopáticos. Fisiopatológicamente se postula que linfocitos $\mathrm{T}$ citotóxicos, mediante interferón $\gamma$ y TNF $\alpha$, inhiben la hematopoyesis y favorecen la apoptosis de células madres hematopoyéticas CD34+. Las manifestaciones clínicas están asociadas a las citopenias. El diagnóstico es de exclusión ${ }^{3}$.

El tratamiento de elección es el trasplante de progenitores hematopoyéticos (TPH) alogénico, donante familiar idéntico (DFI), con tasas de curación en menores de 40 años, sobre $70 \%$. En los pacientes que no tienen donante compatible o mayores de 40 años se utiliza tratamiento inmunosupresor con ciclosporina A e inmunoglobulina antitimocítica de caballo o conejo, con respuesta hematológica a los seis meses entre 45 y $75 \% \%^{4}$.

Durante el embarazo esta condición es aún menos frecuente, hay publicaciones que reportan casos de embarazo y AA que se han recuperado post aborto o post parto y casos de insuficiencia medular preexistentes, que se han agravado du- 
rante el embarazo, lo que sugiere una asociación entre ambas ${ }^{5,6}$.

Estudios muestran alta morbilidad materna y mortalidad en $20-50 \%$ de los casos, asociada a metrorragia peri o post parto, sepsis puerperal y hemorragia intracerebral. El feto puede presentar restricción del crecimiento intrauterino (RCIU), parto prematuro y muerte fetal o neonatal ${ }^{5,7,8,12}$. Debido a la alta mortalidad publicada en series de casos hasta los años '90, autores han recomendado la interrupción del embarazo de forma precoz. Sin embargo, estudios más recientes, con manejo en unidades especializadas han mostrado mejores resultados maternos y perinatales ${ }^{10}$.

La vía de parto ideal no está consensuada, existen series con tasas de hasta $77 \%$ de parto vaginal, con escasas complicaciones maternas, y otras en que se indicó cesárea hasta en $45 \%$. Las series son pequeñas y la mayor parte de la evidencia corresponde a reportes de casos. Algunos autores argumentan que la vía alta es preferida por el alto riesgo de hemorragia cerebral asociada al pujo durante el parto en trombocitopenia severa y por mayor dificultad en el manejo de hemorragia por lesiones del canal de parto, mientras que otros plantean que la vía de parto depende sólo de la indicación obstétrica ${ }^{5,9,10}$.

\section{Caso clínico}

Se presenta el caso de una paciente de 17 años con antecedente de síndrome mielodisplástico hi- poplásico diagnosticado a los 14 años, sin donante para TPH, que abandona controles.

A los tres años del diagnóstico consulta por dos meses de disnea progresiva, epistaxis y gingivorragia. Hemograma: $\mathrm{Hb} 5,2 \mathrm{~g} / \mathrm{dl}$, leucocitos $3.000 /$ ul, RAN $1.833 /$ ul y plaquetas $19.000 /$ ul. $\mathrm{Al}$ examen físico destaca útero grávido, a nivel umbilical. Ecografía confirma 23 semanas de embarazo. Se realiza estudio: mielograma con displasia leve en las tres series, sin blastos, citogenética normal, biopsia de médula ósea con celularidad $<5 \%$ (Figura 1) y ausencia de CD34+, estudio de hemoglobinuria paroxística nocturna con pequeño clon, $0,24 \%$ en eritrocitos y $0,14 \%$ en granulocitos. Se diagnostica como AA severa e inicia ciclosporina y linfoglobulina de caballo. Evoluciona sin complicaciones desde el punto de vista obstétrico, pero sin mejoría de parámetros hematológicos, con necesidad transfusional semanal, para mantener $\mathrm{Hb}>$ a $8 \mathrm{~g} / \mathrm{dl}$ y plaquetas $>20.000 /$ ul. Cabe hacer notar que fue difícil mantener niveles terapéuticos de ciclosporina, debido a un mayor metabolismo durante el embarazo. A las 36 semanas de gestación se hospitaliza e indica metilprednisolona $500 \mathrm{mg} /$ día en bolos, por tres días previos al parto, asociado a transfusión de 15 unidades de plaquetas $1 \mathrm{~h}$ antes del parto para alcanzar niveles mayores a 100.000/ul. Se realiza cesárea por técnica de Joel Cohen, bajo anestesia general, sin incidentes. Recién nacido masculino, peso 2.795 g, Apgar 8-9. La paciente evoluciona satisfactoriamente en puerperio inmediato, requiriendo transfusión de dos unidades de GR 9 h post

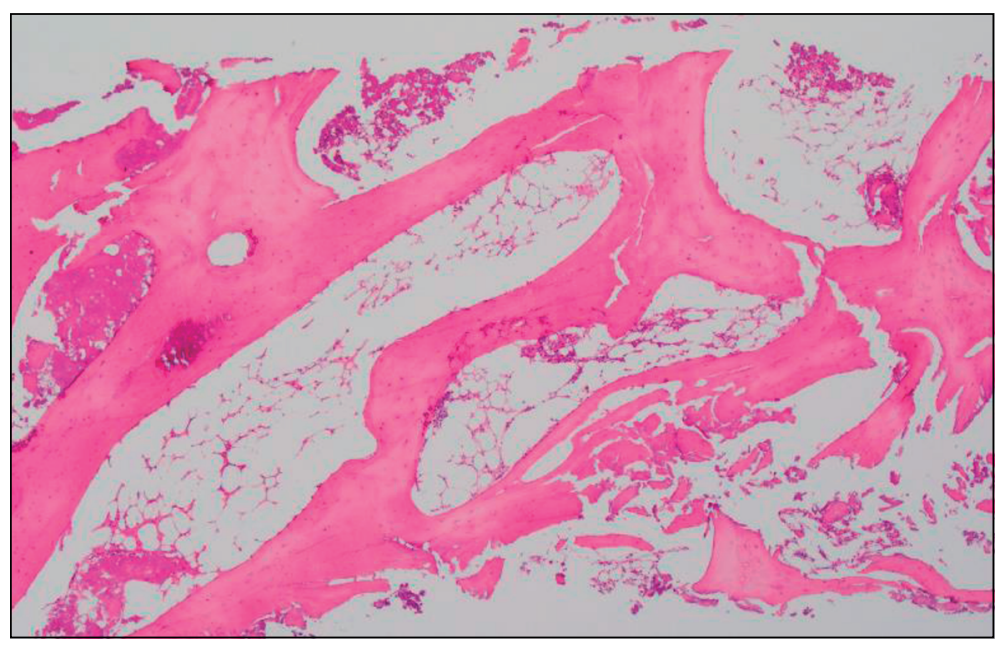

Figura 1. Biopsia de médula ósea. A. Celularidad disminuida para la edad, menor a 5\%, tinción hematoxilina-eosina $4 x$. 
cesárea y 8 unidades de plaquetas al quinto día post operatorio. Se da el alta en buenas condiciones en el octavo día post parto. Se indica medroxiprogesterona acetato $150 \mathrm{mg} / \mathrm{ml}$, una ampolla cada tres meses para manejo anticonceptivo y control de hipermenorrea y bromocriptina para suprimir lactancia. Al control hematológico a los seis meses de la administración de linfoglobulina, mantiene requerimiento trasnfusionales de GR y plaquetas, $\mathrm{Hb} 5,7 \mathrm{~g} / \mathrm{dl}$, RAN 1.192/ul y plaquetas 6.000/ul, Se considera sin respuesta y se programa segundo curso de inmunosupresión.

\section{Discusión}

Se presenta el caso clínico de una adolescente primigesta con aplasia medular severa agravada por embarazo no programado, con resolución favorable.

La paciente presentaba el antecedente de síndrome mielodisplástico/hipoplástico, que luego de 3 años fue catalogado como aplasia medular severa, por la ausencia de progenitores hematopoyéticos en la biopsia de médula ósea, celularidad $<5 \%$ y citogenética normal.

Respecto al tratamiento, el TPH está contraindicado durante el embarazo por requerir altas dosis de inmunosupresión o radioterapia, lo que podría ser tóxica para el feto. El uso de tratamiento inmunosupresor con ciclosporina y globulina antitimocítica son de categoría $\mathrm{C}$ y se han usado en el segundo trimestre de embarazo sin presentar efectos teratogénicos en el feto, su excreción por la leche limita la lactancia. Su uso junto a un apoyo transfusional óptimo son el tratamiento de elección ${ }^{8,9,12,13}$.

Hasta la fecha, no existen reportes nacionales de aplasia medular y embarazo, sólo de tratamiento en pacientes adultos. ${ }^{14}$ Las publicaciones internacionales, son en su mayoría reportes de casos, estableciendo una clara asociación entre aplasia medular y aumento de morbimortalidad materno-fetal ${ }^{5,7,8,12}$.

El trabajo coordinado de hematólogos, obstetras y anestesistas es crucial para llevar a cabo un embarazo y parto con éxito. El apoyo transfusional durante todo el proceso es prioritario para mantener rangos de hemoglobina y plaquetas sobre el umbral de riesgo, British Committee for Standards in Hematology, reco- mienda una $\mathrm{Hb}>8 \mathrm{~g} / \mathrm{dl}$ y plaquetas $>20.000 / \mathrm{ul}$ en el embarazo ${ }^{12}$. Por las múltiples transfusiones recibidas, es importante evaluar complicaciones potenciales como hemosiderosis secundaria y refractariedad plaquetaria, por desarrollo de aloanticuerpos HLA.

Nuestra paciente fue diagnosticada en el segundo trimestre de embarazo, por lo que recibió ciclosporina e inmunoglobulina antitimocítica de caballo, asociado a un control frecuente con apoyo transfusional, además se usó corticoides previo al parto, ya que podría mejorar la respuesta.

La decisión del momento y vía de parto en este caso, se realizó en reunión de especialidades, en este caso se optó por cesárea con anestesia general, por presentar trombocitopenia severa $<10.000 / \mathrm{ul}$, con apoyo transfusional para niveles de $\mathrm{Hb}>8$ $\mathrm{g} / \mathrm{dl}$ y plaquetas $>100.000 / \mathrm{ul}$ con el objetivo de minimizar el riesgo hemorrágico, complicación frecuente y potencialmente mortal, sin embargo, en la mayoría de las publicaciones se ha optado por vía vaginal, con analgesia endovenosa, la anestesia de neuroeje es evitada ${ }^{11,12}$.

El desenlace fue exitoso sin complicaciones maternas ni fetales, a pesar que la paciente no ha presentado respuesta al tratamiento inmunosupresor a los seis meses, por lo que se planificó un segundo curso de inmunosupresión.

\section{Referencias}

1. Young N. Current concepts in the pathophysiology and treatment of aplastic anemia. Educational Program American Society of Hematology 2016.

2. Brodsky R, Jones R. Aplastic anaemia. Lancet 2005; 365: 1647-56.

3. Young N. Current concepts in the pathophysiology and treatment of aplastic anemia. Educational Program American Society of Hematology 2016.

4. Bacigalupo A. How I treat acquired aplastic anemia. Blood 2017; 129 (11): 1428-36.

5. Tichelli A, Socié G, Marsh J, Barge R, Frickhofen N, McCann S, et al. Outcome of pregnancy and disease course among women with aplastic anemia treated with immunosuppression. Ann Intern Med 2002; 137 (3): 164-72.

6. Goldstein IM, Coller BS. Aplastic anemia in pregnancy: recovery after normal spontaneuos delivery. Ann Intern Med 1975; 82: 537-9.

7. Deka D, Malhotra N, Sinha A, Banerjee N, Kashyap R, 
Roy KK. Pregnancy associated aplastic anemia: maternal and fetal outcome. J Obstet Gynaecol Res 2003; 29 (2): 67-72.

8. Choudhry VP, Gupta S, Gupta M, Kashyap R, Saxena R. Pregnancy associated aplastic anemia-a series of 10 cases with review of literature. Hematology 2002; 7 (4): 233-8.

9. Shin JE, Lee Y, Kim SJ, Shin JC. Association of severe thrombocytopenia and poor prognosis in pregnancies with aplastic anemia. PLoS One 2014; 9 (7): 1-6.

10. Marsh JC, Ball SE, Cavenagh J, Darbyshire P, Dokal I, Gordon-Smith EC, et al. Guidelines for the diagnosis and management of aplastic anaemia. Br J Haematol 2009; 147 (1): 43-70.

11. Riveros-Pérez E, Hermesch A, Barbour L, Hawkins J.
Aplastic anemia in two consecutive pregnancies: Obstetric and anesthetic management. International Journal of Obstetric Anesthesia. 2017 August. DOI: http://dx.doi. org/10.1016/j.ijoa.2017.08.010.

12. Bo L, Mei-Ying L, Yang Z, Shan-Mi W, Xiao-Hong Z. Aplastic anemia associated with pregnancy: maternal and fetal complications. J Matern Fetal Neonatal Med 2016; 29 (7): 1120-4.

13. Stibbe KJ, Wildschut HI, Lugtenburg PJ. Management of aplastic anemia in a woman during pregnancy: a case report. J Med Report 2015; 5: 66.

14. Cabrera ME, Pinto D. Anemia aplástica adquirida del adulto. Su tratamiento a propósito de dos casos. Rev Med Chile 2003; 131: 1439-43. 